\title{
Electronic nose with polymer-composite sensors for monitoring fungal deterioration of stored rapeseed $* *$
}

\author{
Marek Gancarz", Jolanta Wawrzyniak², Marzena Gawrysiak-Witulska ${ }^{2}$, Dariusz Wiqcek ${ }^{1}$, \\ Agnieszka Nawrocka ${ }^{1}$, and Robert Rusinek ${ }^{1 *}$ \\ ${ }^{1}$ Institute of Agrophysics Polish Academy of Sciences, Doświadczalna 4, 20-290 Lublin, Poland \\ ${ }^{2}$ Institute of Plant Origin Food Technology, Faculty of Food Science and Nutrition, Poznań University of Life Science, \\ Wojska Polskiego 28, 60-637 Poznań, Poland \\ Received May 30, 2016; accepted May 30, 2017
}

\begin{abstract}
A b s t r a c t. Investigations were performed to examine the possibility of using an electronic nose to monitor development of fungal microflora during the first eighteen days of rapeseed storage. The Cyranose 320 device manufactured by Sensigent was used to analyse volatile organic compounds. Each sample of infected material was divided into three parts and the degree of spoilage was measured in three ways: analysis of colony forming units, determination of ergosterol content, and measurement of volatile organic compounds with the e-nose. Principal component analysis was performed on the generated patterns of signals and six groups of different spoilage levels were isolated. An analysis of sensorgrams for a few sensors with a strong signal for each group of rapeseed spoilage was performed. The ratio of the association time to the steady state was calculated. This ratio was different for the low level and the highest level of ergosterol and colony forming units. The results have shown that the e-nose can be a useful tool for quick estimation of the degree of rapeseed spoilage.

K e y w o r d s: principal component analysis, e-nose, rapeseed, ergosterol, colony forming units, volatile organic compounds
\end{abstract}

\section{INTRODUCTION}

Currently, rapeseed accounts for approximately $11 \%$ of the global production of oil-bearing plants (Rusinek et al., 2012). Improvement of processing and storage conditions is very important in order to get a high quality product. Rapeseed stored in silos is at constant risk of deterioration of its quality. Despite application of appropriate preparatory treatments, rapeseeds, unlike cereal grains, are more susceptible to damage and deterioration due to the high fat content, which increases the intensity of unfavourable biological and chemical processes in damaged seeds (Rusinek et al., 2009; Rusinek and Kobyłka, 2014; Rybiński et al.,

\footnotetext{
*Corresponding author e-mail: r.rusinek@ipan.lublin.pl

**This work was supported by the National Centre for Research Development (NCRD), Grant No. PBS2/A8/22/2013.
}

2009). Microbial activity increases in damaged seeds, which leads to reduction of the quality or even destruction of the raw material. These phenomena are particularly dangerous in moist seeds, which are excessively loaded or stored at too high temperatures with high local gradients resulting from insufficient seedbed aeration. These biological processes may be initiated very fast, sometimes within a few days after filling the silo.

Numerous methods are in use for detection of spoilage of stored seeds. Jeleń and Wąsowicz (1998) reviewed proposed methods of detection and enumeration of fungal microflora in stored grains and concluded that all of them suffer some drawbacks. All microbiological methods giving an answer about the presence of fungi and fungal contamination levels are laborious and time-consuming. On the other hand, there are indirect methods for detection and quantification of fungal infection in grain based on assessment of chemical markers such as ergosterol, chitin, or adenosine triphosphate (Gawrysiak-Witulska et al., 2008). However, these methods require demanding and laborious sample preparation (Abramson and Smith, 2003; Magan and Evans, 2000; Olsson et al., 2000). Smelling the grain for detection of its quality by man is another option, but this constitutes a potential health hazard through inhalation of mould spores and mycotoxins. Therefore, application of an electronic nose (e-nose) for the analysis of the quality of grain is considered as a promising and quick method (Sung et al., 2014). The technique was developed at the end of the last century (Olsson et al., 2000; Oshita et al., 2006). At the current stage of evolution of sensor technology, a device like the e-nose offers new possibilities not

(C) 2017 Institute of Agrophysics, Polish Academy of Sciences 
only for assessment of grain quality but generally in food (Loutfi et al., 2015), cosmetic, or pharmaceutical industries, as well as in environment control and clinical disease diagnosis (Casalinuovo et al., 2006; Guz et al., 2015; Pathange et al., 2006; Peris and Escuder, 2009).

Odours and volatiles are produced by a wide range of organisms including those causing deterioration of plant granular materials (Presicce et al., 2006). Volatile metabolites produced by fungi during the spoilage process can be characteristic markers of fungal presence. Fungi produce a unique odour of many fermented products (Perkowski et al., 2008, 2012). Magan and Evans (2000) have reviewed volatile compounds found in cultures of fungi grown on different grain and cereal substrates. Their work has shown a wide range of volatiles produced by fungi e.g. aldehydes, ketones, alcohols etc. There is potential for distinguishing between species of fungi based on characteristic volatile substances. The authors suggest that it may be possible to use the e-nose to try to distinguish them. Studies using the e-nose for quality grading of barley grain have been reported by Olsson et al. (2000). The authors used an electronic nose and gas chromatography combined with mass spectrometry (GC-MS) to quantify the level of ergosterol and colony forming units ( $\mathrm{CFU}$ ) of naturally contaminated barley samples. A conclusion was drawn that both methods could be used to discriminate between normal and offodour grain volatiles and to predict ergosterol levels in barley. Zhang and Wang (2007) efficiently used an e-nose to evaluate insect damage and classify the storage age of stored wheat. The results analysed by principal component analysis and linear discriminant analysis provided a good classification in the storage age. Regarding insect damage, all wheat samples were distinguished properly. Zhou et al. (2012) used an electronic nose to distinguish between three varieties of wheat seeds. According to the authors, gas chromatography-mass spectrometry analysis of the headspaces of the same samples confirmed that the e-nose was a powerful device to identify the variety of wheat. However, up to now there have been not many reports on successful evaluation of spoilage of oil seed using the electronic nose system (Kubiak, 2012, 2014). Therefore, the objective of the present study was to compare e-nose responses to volatiles produced by stored rapeseed with CFU counts and ergosterol levels in the early stage of spoilage for up to 18 days after filling the silo.

\section{MATERIAL AND METHODS}

The examined material was winter high-oleic rapeseed with fat content of approximately $43 \%$, harvested in Poland, provided by the Institute of Agrophysics PAS (Poland). Rapeseed samples with different levels of spoilage were prepared by storing the rapeseed in a conditioning chamber at constant conditions of seed moisture content $(12 \%$ w.b. $)$ and temperature $\left(30^{\circ} \mathrm{C}\right)$, as described earlier by Wawrzyniak et al. (2013). The chamber had been used previously to study the changes in the quality of grain and rapeseed during storage (Gawrysiak-Witulska et al., 2011, 2012). Six spoiled samples were selected every three days during 18 days of storage including a control sample (Table 1). Each sample was divided into three $200 \mathrm{~g}$ parts for analysis of ergosterol levels, fungal CFU, and responses to volatiles using the e-nose.

The level of mould contamination of the rapeseed samples was determined as Colony Forming Units (CFU) of moulds per $1 \mathrm{~g}$ of grain. Analysis of CFUs in the rapeseed was performed in accordance with the procedure described in the standard (PN-ISO 21527-2:2009).

Rapeseed samples (10 g) were placed in $90 \mathrm{ml}$ sterilized Phosphate Buffered Saline (PBS) containing a wetting agent Tween $80\left(0.003 \mathrm{~g} \mathrm{l}^{-1}\right)$ and, after soaking for $30 \mathrm{~min}$, the samples were shaken for $2.5 \mathrm{~min}$. During microbiological analyses, serial dilutions were prepared following PN-EN ISO 6887-1:2017-05. $1 \mathrm{ml}$ samples were spread onto duplicate sterilized plates and covered with YGC Agar containing glucose, agar, yeast extract, and chloramphenicol $\left(100 \mu \mathrm{gl}^{-1}\right)$. After 3 days of incubation, the CFUs of moulds were evaluated using a colony counter. All treatments were performed in triplicate.

The ergosterol extraction was carried out according to the method described by Abramson and Smith (2003) and Pronyk et al. (2006). Briefly, $5 \mathrm{~g}$ rapeseed samples were milled, extracted in $50 \mathrm{ml}$ of methanol, cooled, filtered, and saponified for $30 \mathrm{~min}$. After saponification, the obtained samples were cooled and filtered once again. Next, the mixture was extracted twice with $50 \mathrm{ml}$ of $\mathrm{n}$-hexane. $12.5 \mathrm{ml}$ of the n-hexane extract was taken to dryness under a nitrogen stream at $40^{\circ} \mathrm{C}$. The obtained residue was dissolved in $2 \mathrm{ml}$ of cyclohexane, added to a $3 \mathrm{ml} \mathrm{SPE} \mathrm{(solid} \mathrm{phase} \mathrm{extrac-}$ tion) cartridge containing $500 \mathrm{mg}$ silica gel (Strat Silica, Phenomenex), and rinsed with cyclohexane. To obtain ergosterol acetate, acetylation by acetic anhydride was required. After acetylation, the eluate was taken to dryness. For HPLC analysis, the purified ergosterol acetate was dissolved in $1 \mathrm{ml}$ of acetonitrile. $10 \mathrm{ml}$-aliquots were injected into an HPLC system (Acella, Thermo Scientific, USA) with a C-18 reverse-phase column (Hypersil Gold, 50 x $2.1 \mathrm{~mm}, 1.9 \mathrm{~mm}$ particles, Thermo Scientifc, USA) at temperature $35^{\circ} \mathrm{C}$. The diode-array spectrophotometric detector was set at $282 \mathrm{~nm}$. Methanol at $450 \mathrm{ml} \mathrm{min}^{-1}$ was used as the mobile phase. Ergosterol acetate was eluted at 2.15 min (Gancarz et al., 2017).

The Cyranose 320 is a fully integrated hand-held chemical vapour sensing instrument designed specifically to detect and identify complex chemical mixtures that constitute aromas, odours, fragrances, formulations, spills, and leaks. It is also used to identify simple mixtures and individual chemical compounds (Casalinuovo et al., 2006). Model 320 has 32 polymer-composite sensors. The conductivity of the polymer changes when molecules are absorbed at the conducting particle chain. The sensors strongly respond 


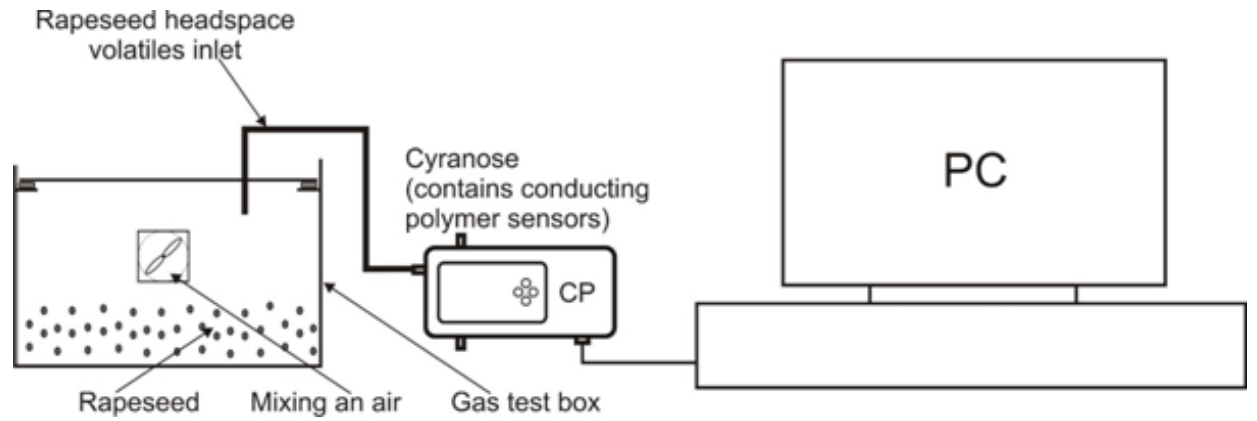

Fig. 1. Experimental setup for the e-nose for headspace volatile sampling.

to the presence of ketones, fatty acids, esters, and alcohols that can be expected in spoilage rapeseed. Typical response by the sensors is represented by 32 curves of conductivity against time (sensorgram) when the volatiles from the rapeseed reach the measurement chamber.

The measurements of volatile organic compounds (VOC) were carried out using an experimental set presented in Fig. 1. Each sample of rapeseed was placed into a special Gas Test Box (Figaro, Japan). A measurement cycle according to the sample protocol consisted of 10 $\mathrm{s}$ baseline purge, $40 \mathrm{~s}$ sample draw-in, $5 \mathrm{~s}$ laboratory air purge, not less than $120 \mathrm{~s}$ sample purge. The temperature of the active surface of the sensors during the measurement of volatile substances was $42^{\circ} \mathrm{C}$ for optimum performance of the e-nose during data acquisition. The mean pump speed for the baseline and sample draw-in was $150 \mathrm{ml} \mathrm{min}^{-1}$ while the high pump speed for sample purge was $300 \mathrm{ml} \mathrm{min}^{-1}$.

A data matrix with 60 rows and 32 columns was built for six samples and 20 rows and 32 columns for overcoming the inability of the e-nose to distinguish between the species of spoiled and unspoiled seed. PCA were the pattern-recognition techniques used. The set of the sensor data obtained was analysed using a 'Canonical' pattern recognition algorithm. This model was the most appropriate for these applications based on manufacturer guidelines.

\section{RESULTS AND DISCUSSION}

The Cyranose was trained to differentiate between six samples of spoiled rapeseed and the control sample. The device showed great sensitivity to the presence of the volatile chemical compounds elicited from spoiled rapeseed. Each line in Fig. 2 represents a response of the individual sensor. The vertical axis represents sensor response:

$$
\frac{\Delta R}{R_{0}}=\frac{R_{\max }-R_{0}}{R_{0}},
$$

were: $R_{0}$-resistance at baseline, $R_{\max }-$ maximum resistance.

This response suggests that the electronic nose could be used successfully for detection of volatile substances in spoiled rapeseed (Henderson et al., 2010). Similar research

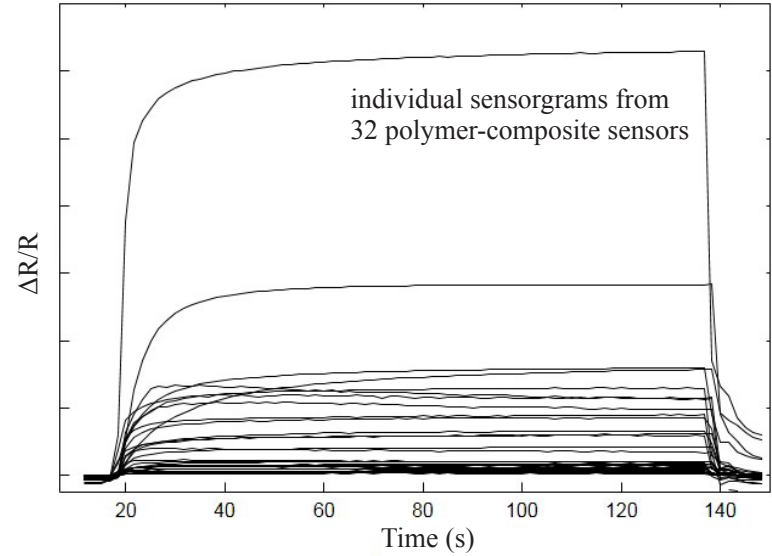

Fig. 2. Example of response of Cyranose to volatiles from a spoiled sample of rapeseed.

has been successfully conducted by Olsson et al. (2000) for barley. The authors concluded that it was possible to quantify ergosterol and CFU using the electronic nose.

The first step of the e-nose calibration was to overcome the ability of Cyranose to distinguish between species of spoiled and unspoiled seed. In this case, the analysis revealed the presence or absence of spoilage signs in the samples (the control sample - ergosterol less than $1 \mathrm{mg} \mathrm{g}^{-1}$ $v s$ the spoiled sample - ergosterol more than $6 \mathrm{mg} \mathrm{g}^{-1}$ ). This method gave two very distinct groups in the PCA 2D plot with $100 \%$ correct cross-validation. This test revealed that Cyranose distinguished spoiled from unspoiled samples of rapeseed (Fig. 3). The data obtained from 32 sensors gave information that the two principal components (PC1 85\% and PC2 15\%) accounted for our desired $100 \%$ of total variation.

Henderson et al. (2010) and Panigrahi et al. (2006) suggested switching off a few sensors that were sensitive to water vapour. In our research, we performed two kinds of tests with 32 and 28 sensors (except for 4 that were sensitive to water vapour), but clearer and more stable results were obtained with 32 sensors (Fig. 4). Additionally, all rapeseed samples had the same moisture content during the tests. Figure 4 shows a typical pattern of responses (smellprint) from the sensors reacting to volatiles from the headspace of the control and spoiled samples. These 


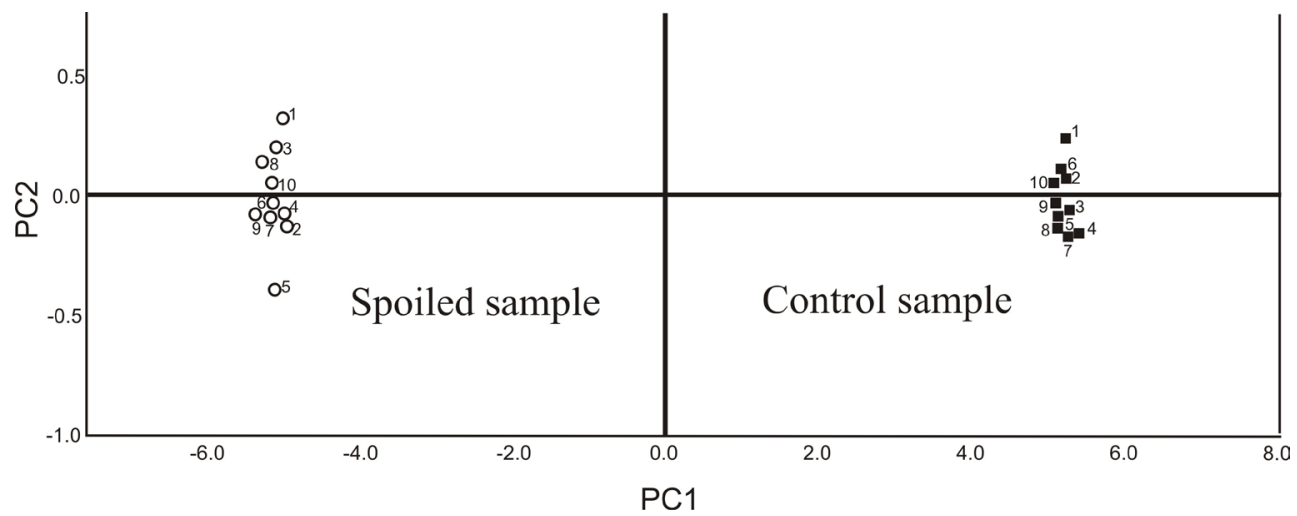

Fig. 3. Principal component analysis describing discrimination between control and spoiled samples.
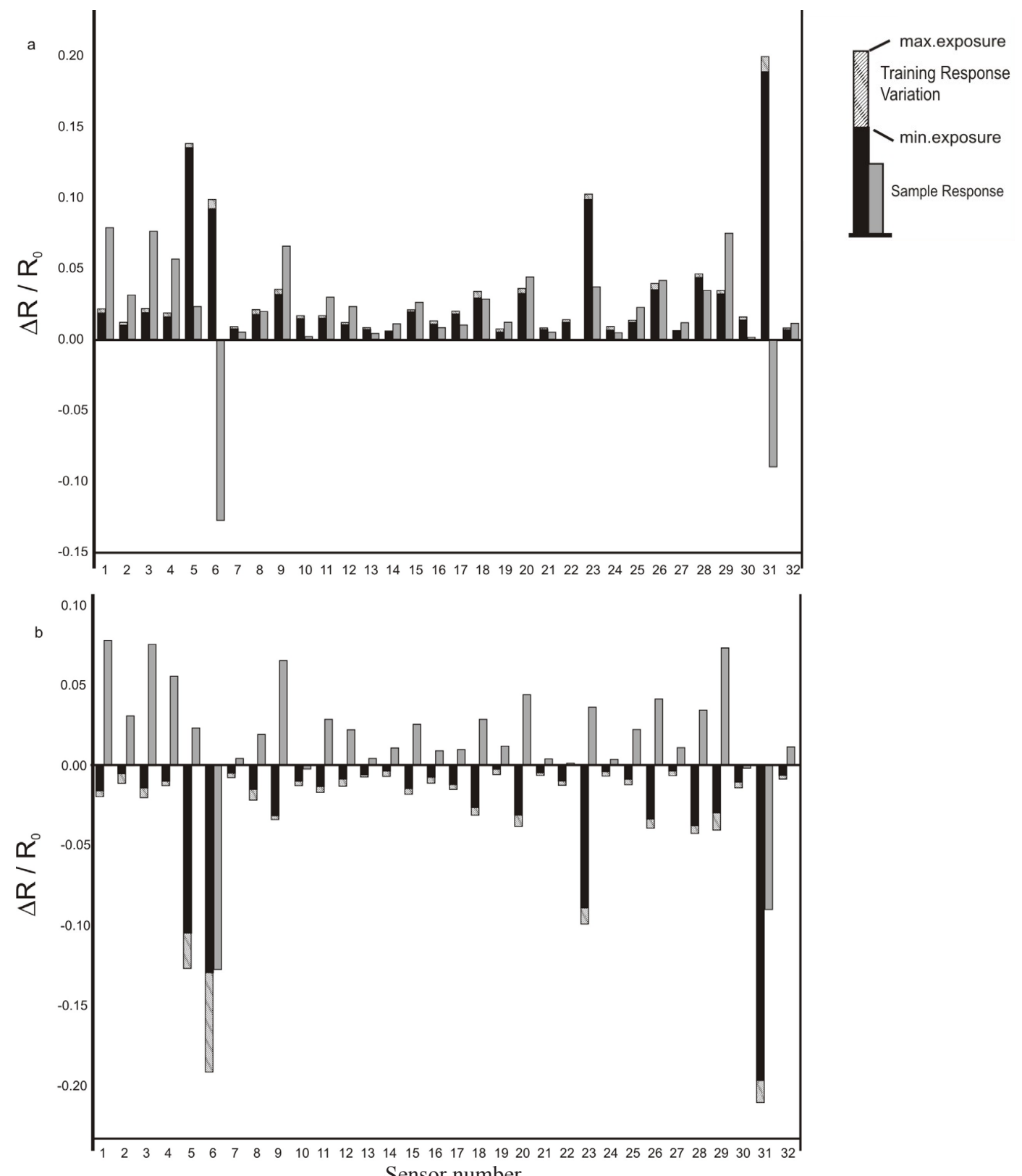

Fig. 4. Typical smellprint from the 32 sensors reacting to volatiles from the headspace for: $a-$ spoiled and b-control samples. 
T a b l e 1. Contents of ergosterol (ERG) and colony forming units (CFU)

\begin{tabular}{cccl}
\hline $\begin{array}{c}\text { Sample number } \\
(\text { days of storage) }\end{array}$ & $\begin{array}{c}\text { Ergosterol } \\
\left(\mu \mathrm{g} \mathrm{g}^{-1}\right)\end{array}$ & $\begin{array}{c}\text { Colony forming units } \\
\left(\log _{10} \mathrm{cfu}^{-1}\right)\end{array}$ & Fungal microflora \\
\hline control & $0.73 \pm 0.07$ & $2.25 \pm 0.35$ & Mucor, Acremonium, Cladosporium \\
$1(3)$ & $7.54 \pm 0.07$ & $2.42 \pm 0.03$ & Mucor, Acremonium, Cladosporium, Fusarium \\
$2(6)$ & $8.72 \pm 0.06$ & $3.50 \pm 0.01$ & Mucor, Acremonium, Cladosporium \\
$3(9)$ & $10.17 \pm 0.08$ & $3.88 \pm 0.17$ & Eurotium, Penicillium, (marginally: Mucor, Cladosporium) \\
$4(12)$ & $10.31 \pm 0.11$ & $4.13 \pm 0.04$ & Eurotium, Penicillium \\
$5(15)$ & $8.34 \pm 0.13$ & $4.31 \pm 0.04$ & Eurotium, Penicillium \\
$6(18)$ & $10.77 \pm 0.10$ & $4.39 \pm 0.11$ & Eurotium, Penicillium \\
\hline
\end{tabular}

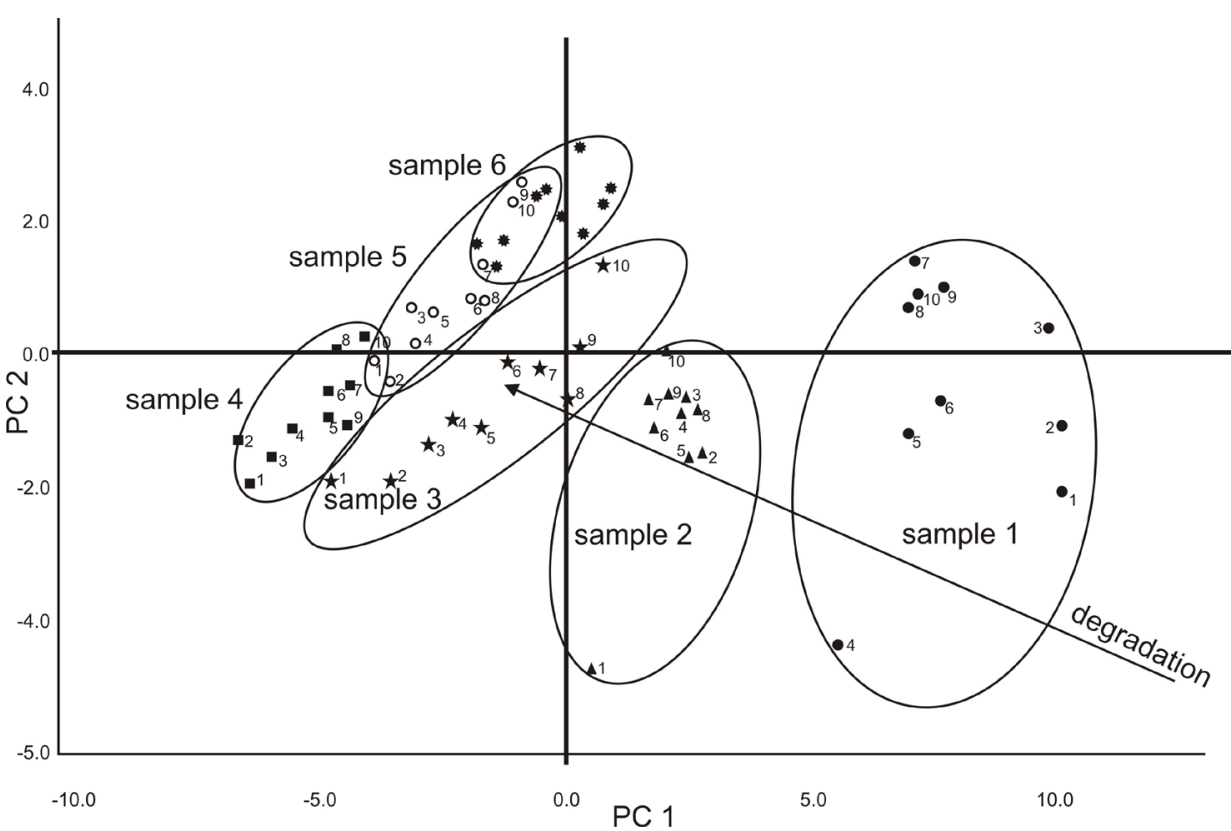

Fig. 5. PCA plot for rapeseed degradation.

two smellprint graphs clearly show the differences in the responses of the individual sensors. In this research, each sample was marked by an individual smellprint.

The concentrations of ergosterol and CFUs of moulds in the samples were determined after eighteen days of storage and they are presented in Table 1. PCA was used to investigate how well the e-nose analysis separated the levels of seed spoilage for over a dozen days of storage in unfavourable conditions. The principal component analysis plot (Fig. 5) gave discrete grouping with 85\% correct and another $15 \%$ incorrect cross-validation. Despite some minor differences in the CFU and ergosterol content, the e-nose provided responses according to the level of spoilage (Table 1). The ergosterol content as well as the CFU for sample 1 (3 days of storage), 2 (6 days of storage), and 3 (9 days of storage) clearly increased. The ergosterol content for samples 4 and 6 was at a similar level and below that level in the case of sample 5 (Fig. 5). Balasubramanian et al. (2007) reported similar results for three samples of barley stored for 20 days, namely, clean barley, naturally Fusarium-infected barley, and Fusarium-inoculated clean barley. Based on e-nose analysis, the authors were able to classify the samples of barley into two groups: good (ergosterol $\leq 3 \mathrm{mg} \mathrm{g}^{-1}$ ) and bad (ergosterol $\geq 3 \mathrm{mg} \mathrm{g}^{-1}$ ) with accuracy up to $91 \%$. It should be added that an ergosterol concentration of $3 \mathrm{mg} \mathrm{g}^{-1}$ is the maximum concentration of ergosterol that can be found in cereal grains intended for human consumption. However, the ergosterol level in plant materials is dependent on the growth matrices (Wawrzyniak and Waśkiewicz, 2014) and in the case of rapeseed, the limit of the ergosterol concentration characterizing seeds with good quality is higher and should not exceed $6 \mathrm{mg} \mathrm{g}^{-1}$. In our experiment, the ergosterol concentration increased with the increasing storage time from 7.5 to $10.77 \mathrm{mg} \mathrm{g}^{-1}$ (Table 1 ). In all samples, the ergosterol concentration exceeded the level of $6 \mathrm{mg} \mathrm{g}^{-1}$, while the CFU g ${ }^{-1}$ 


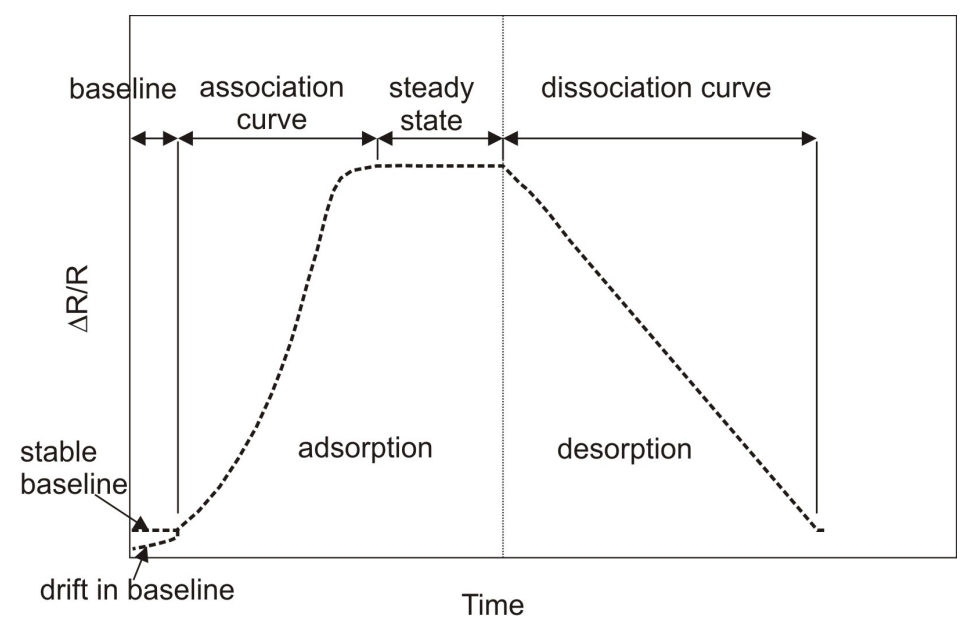

Fig. 6. Scheme of sensorgram of polymer-composite sensors.

a

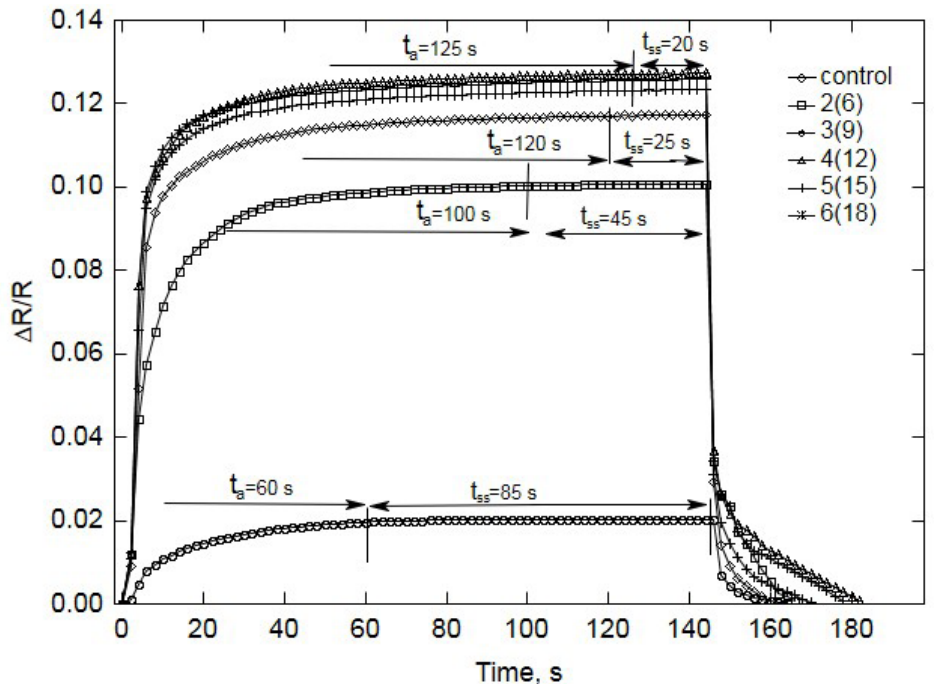

$\mathrm{b}$

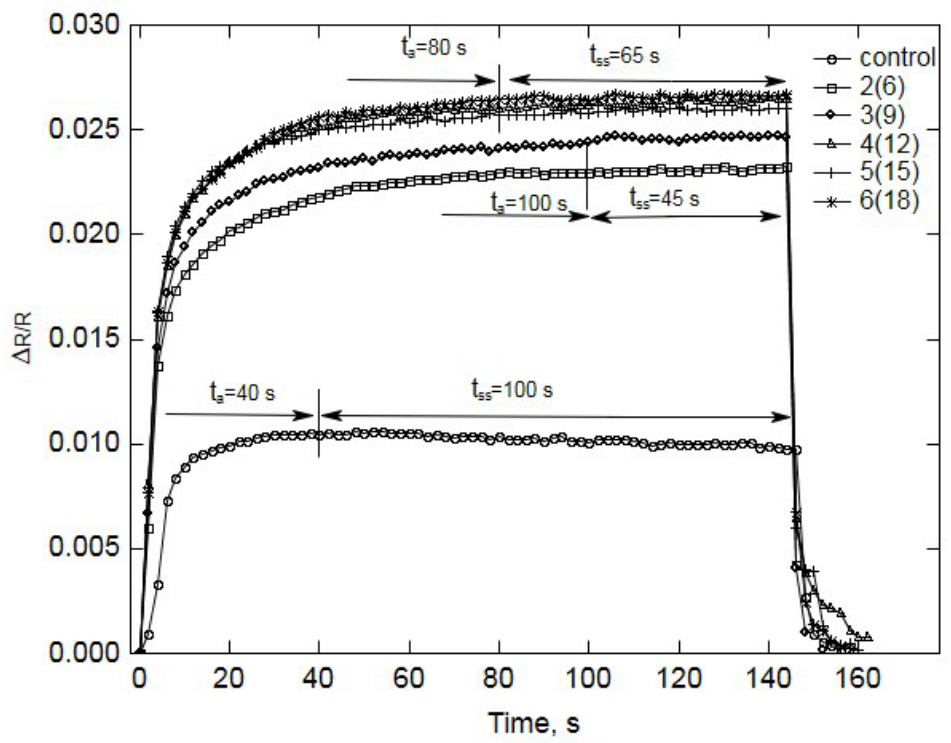

Fig. 7. Sensorgrams of sensor: $\mathrm{a}-31$ and $\mathrm{b}-28$; for 18 days of storage (association time $\left(\mathrm{t}_{\mathrm{a}}\right)$, steady state $\left(\mathrm{t}_{\mathrm{ss}}\right)$ ). 
reached a level slightly above 4 . The growth and stability of both parameters was reflected in the results of the e-nose research and PCA analysis.

The PCA plot presented in Fig. 5 clearlyshows three different groups of data for samples 1,2 , and 3 . The CFU and ergosterol concentration increased during the first nine days of storage (Table 1). This tendency was clearly reflected by the electronic nose measurement as three distinct groups (samples 1, 2, and 3). During the last days of storage represented by samples 4,5 , and 6 , the ergosterol concentration did not change markedly. The electronic nose results shown in figure 5 present this effect as three overlapping groups.

The PCA plot in Fig. 5 shows that the data for seeds of the worst quality are more tightly clustered and located at left side of vertical axis, while the data for good quality seeds are located on the right side of axis. A similar trend was observed in Fig. 3. Generally, the analysis has shown clear groups of data corresponding to six samples as well as a trend that is indicated in Fig. 5 by the arrow pointing to the direction of the increasing degradation of seeds. Additionally, the authors suggested that the distinct grouping of sample 1,2, and 3 was correlated mainly with field microflora and the overlapping groups of sample 4, 5, and 6 were correlated primarily with storage microflora (Gancarz et al., 2017).

Each smellprint was built from individual response of 32 polymer-composite sensors (Balasubramanian et al., 2007). Sensor response is a curve called a sensor drift or a sensorgram. Generally, it consists of two phases, i.e. adsorption and desorption. In this work, the authors analysed the sensorgrams of several best-responsive sensors of VOCs from spoiled rapeseed. Several factors can be analysed: the baseline (with drift or stable), association curve, steady state (horizontal curve, if obtained), or the dissociation curve (Fig. 6).

Literature shows similar analyses but mostly done for chemical volatiles. For example, Mielle and Marquis (2000) analysed the sensor drift for an ethanol concentra-

T a b l e 2. Ratio of the association time curve $t_{a}$ to the steady state $\mathrm{t}_{\mathrm{ss}}$

\begin{tabular}{cccccc}
\hline & \multicolumn{5}{c}{ Sensor number } \\
\cline { 2 - 6 } $\begin{array}{c}\text { Sample } \\
\text { number (days } \\
\text { of storage) }\end{array}$ & 5 & 6 & 26 & 28 & 31 \\
\cline { 2 - 6 } & 0.31 & 0.41 & 0.28 & 0.40 & 0.70 \\
\hline control & 0.55 & $*$ & 2.47 & 2.22 & 2.22 \\
$2(6)$ & 1.04 & $*$ & 2.47 & 2.22 & 4.80 \\
$3(9)$ & 0.75 & $*$ & 2.47 & 1.23 & 6.25 \\
$4(12)$ & 0.84 & $*$ & 2.47 & 1.23 & 6.25 \\
$5(15)$ & 0.84 & $*$ & 2.47 & 1.23 & 6.25 \\
$6(18)$ & & & & & \\
\hline
\end{tabular}

tion. A sensory response feedback process was discussed by Zhang et al. (2008) for a few chemical volatiles, and Garcia-Gonzalez and Aparicio (2010) for volatile compounds of virgin olive oil.

For this purpose, 32 sensorgrams of Cyranose sensors were analysed. Only 15 of them gave a clear and responsive signal on spoiled rapeseed VOCs. These were the following sensors: $1,2,3,4,5,6,8,9,18,20,23,26,28,29$, and 31 (Figs 2, 4a and 4b). Analysis of association time the steady state and dissociation were analysed for a few sensors with a strong signal from this group. Sensorgrams 28 and 31 were chosen to be presented in this paper.

Cyranose was built of 32 polymer-composite sensors with a conducting particle chain. In this type of sensors, the conducting particle chain reacts to VOCs and produces a change in DC resistance. These responses can be different for different substances (Gruber et al., 2013). The concentration of VOCs from fungi increased with CFU and ergosterol content (ERG) at the time of storage (Table 1). Some polymer sensor response from this group generally correlated with this trend (Fig. 7), especially for the first 9 days of the experiment when fungi characteristic for field microflora dominated (Fusarium, Mucor, Acremonium, Cladosporium). The next three groups of response of the sensors were similar (sample 4, 5, and 6) and, based on the analysis of microflora, can be correlated with another type of fungi (10-18 days). In this period, storage microflora dominated (Penicillium, Eurotium). A similar trend is also observed in the PCA analysis presented in Fig. 5. The last three points of samples 4,5 , and $6(12,15$, and 18 days) were located next to each other but clearly differed form samples 1,2 , and 3 .

The sensorgram showed that the dissociation time was very short; therefore, it was not analysed. Quick refresh is a characteristic feature of this kind of sensor. The ratio of the association time to the steady state gave quick information about the quality state. This ratio was different for the low level and the highest level of ERG. Each sensor gave low response in the control sample, and the steady state was quickly reached. The ratio of the association time $\left(t_{a}\right)$ to the steady state $\left(\mathrm{t}_{\mathrm{ss}}\right)$ was less than one (Table 2 ).

A characteristic feature of sensor 6 was failure to reach the steady state during taking the sample for $140 \mathrm{~s}$ in this research, although this time was sufficient for the other sensors to reach the steady state. Differences between the responses of sensors depend on individual chemical characteristic of the thin film. Each polymer sensor is chemically unique and absorbs VOCs to a different degree, thus creating a pattern of responses differing from each other.

\section{CONCLUSIONS}

1. The electronic nose with polymer-composite sensors under laboratory conditions distinguished between species of spoiled and unspoiled seeds. Despite some minor differ- 
ences in the colony forming units and ergosterol content, the electronic nose provided responses correctly corresponding to the level of spoilage.

2. Only 15 from the 32 polymer-composite sensors gave a clear and responsive signal on spoiled rapeseed volatile organic compounds. The ratio of the association time to the steady state was different for the control and spoiled samples. This factor and the sensorgram analysis, besides statistical analysis, can describe the quality of seeds quickly and simply.

3. The principal component analysis has shown that the distinct grouping of sample 1, 2, and 3 was correlated mainly with field microflora and the overlapping groups of sample 4,5 , and 6 were correlated primarily with storage microflora. Therefore, the main conclusion from the study is that the electronic nose is a promising tool for quick and non-destructive detection of the level of oil seed spoilage.

4. The present results have indicated that the e-nose can be used to determine rapeseed quality and distinguish fungal species. For these reasons, gas chromatography (GC-MS) is planned to be used to detect particular volatile organic compounds and assign them to the particular fungal species. Moreover, an electronic nose with metal oxide sensors will be used in these studies.

Conflict of interest: The Authors do not declare conflict of interest.

\section{REFERENCES}

Abramson D. and Smith D.M., 2003. Determination of ergosterol In canola (Brassica napus L.) by liquid chromatograph. J. Stored Product Res., 39, 185-191.

Balasubramanian S., Panigrahi S., Kottapalli B., and WolfHall C.E., 2007. Evaluation of anartificial olfactory system for grain quality discrimination. LWT, 40, 1815-1825.

Casalinuovo I. A., Di Pierro D., Coletta M., and Di Fracesco P., 2006. Application of electronic nose for disease diagnosis and food spoilage detection. Sensors, 6, 1428-1439.

PN-EN ISO 6887-1:2017-05. Microbiology of food and animal feeding stuffs - Preparation of test samples, initial suspension and decimal dilutions for microbiological examination - Part 1: General rules for the preparation of the initial suspension and decimal dilutions

Gancarz M., Wawrzyniak J., Gawrysiak-Witulska M., Wiącek D., Nawrocka A., Tadla M., and Rusinek R., 2017. Application of electronic nose with MOS sensors to prediction of rapeseed quality. Measurement, 103, 227-234.

Garcia-Gonzalez D. and Aparicio R., 2010. Coupling MOS sensors and gas chromatography to interpret the sensor responses to complex food aroma: Application to virgin olive oil. Food Chemistry 120, 572-579.

Gawrysiak-Witulska M., Siger A., Wawrzyniak J., Nogalaand Kalucka M., 2011. Changes in tocochromanol content in seeds of Brassica napus L. during adverse conditions of storage. J. Am. Oil Chem. Soclub. JAOCS, 88, 1379-1385, DOI 10.1007/s11746-011-1793-0.

Gawrysiak-Witulska M., Rudzińska M., Wawrzyniak J., and
Siger A., 2012. The effect of temperature and moisture content of stored rapeseed on the phytosterol degradation rate. J. Am. Oil Chem.Soclub. JAOCS, 89, 1673-1679, DOI 10.1007/s11746-012-2064-4 (10\%?).

Gawrysiak-Witulska M., Wawrzyniak J., Ryniecki A., and Perkowski J., 2008. Relationship of ergosterol content and fungal contamination and assessment of technological quality ofmaltingbarley preserved in a metal silo using the near-ambient method. J. Stored Product Res., 44: 360-365.

Gruber J., Nascimento H.M., Yamauchi E.Y., Li R.W.C., Estaves C.H.A., Rehder G.P., Gaylarde C.C., Shirakawa M.A., 2013. A conductive polymer based electronic nose for early detection of Penicillium digitatum in post-harvest oranges. Materials Sci. Eng. C, 33, 2766-2769.

Guz L., Lagód G., Jaromin-Gleń K., Suchorab Z., Sobczuk H., and Bieganowski A., 2015. Applicationof gas sensor arrays in assessment of wastewater purification effects. Sensors, 15, 1-21.

Henderson W.G., Khalilian A., Han Y.J., Greene J.K., and Degenhardt D.C., 2010. Detecting stink bugs/damage in cotton utilizing a portable electronic nose. Computers Electronics in Agric., 70 157-162.

Jeleń H. and Wąsowicz E., 1998. Volatile fungal metabolites and their relation to the spoilage of agricultural commodities. Food Review Int., 14(4), 391-426.

Kubiak A., 2012. Evaluation of the quality of postharvest rapeseed by means of an electronic nose. J. Sci. Food Agric., 92(10): 2200-6. DOI:10.1002/jsfa.5613

Kubiak A., 2014. Colorimetric Sensor Arrays for Screening Control of Rapeseed Quality, Sensor Letters, 12(6-7), 1070-1075(6).

Loutfi A., Coradeschi S., Kumar Mani G., Shankar P., and Bosco Balaguru Rayappan J., 2015. Electronic noses for food quality: J. Food Eng., A review, 144, 103-111.

Magan E. and Evans P., 2000. Volatiles as an indicator of fungal activity and differentiation between species, and the potential use of electronic nose technology for early detection of Grain spoilage. J. Stored Products Res., 36, 319-340.

Mielle P. and Marquis F., 2000. Gas sensors arrays (Electronic nose): a study about the speed/accuracy ratio. Sensors Actuators, B, 68, 9-16.

Olsson J., Borjesson T., Lundstedt T., and Schnurer J., 2000. Volatiles for mycological quality grading of barley grains: determinations using gas chromatography-mass spectrometry and electronic nose. Int. J. Food Microbiol., 59, 167-178.

Oshita S., Arase H., Seo Y., Kawagoe Y., Haruta K., Amitani H., Kawana S., and Makino Y., 2006. Odor recognition of soy souce by semiconducting polimer sensor. Trans. ASABE, 49(6), 1839-1844.

Panigrahi S., Balasubramanian S., Gu H., Logue C., and Marchelo M., 2006. Neutral-network-integrated electronic nose system for identification of spoiled beef. LWT, 39, 135-145.

Pathange L.P., Mallikarjunan P., Marini R.P., Keefe S.O., and Vaughan D., 2006. Non-destructive evaluation of apple maturity using an electronic nose system. J. Food Eng., 77, 1018-1023.

Peris M. and Escuder-Gilabert L., 2009. A 21st century for food control: Electronic noses. AnalyticaChimica Acta, 638, $1-15$. 
Perkowski J., Buśko M., Chmielewski J., Góral T., and Tyrakowska B., 2008. Content of Trichodieneand analysis of fungal volatiles (electronic nose) in wheat and triticale grain naturally infected and inoculated with Fusarium culmorum. Int. J. Food Microbiol., 126, 127-134.

Perkowski J., Stuper K., Buśko M., Góral T., Kaczmarek A., and Jeleń H., 2012. Differences in metabolomic profiles of the naturally contaminated grain of barley, oats and rye. J. Cereal Sci., 56, 544-551.

PN-ISO 21527-2: 2009. Microbiology of food and feeding stuffs - Horizontal method for the enumeration of yeasts and moulds - Part 2: Colony count technique in products with water activity less than or equal to 0.95 .

PN-EN ISO 6887-1: 2017-05 Microbiology of food and animal feeding stuffs - Preparation of test samples, initial suspension and decimal dilutions for microbiological examination - Part 1: General rules for the preparation of the initial suspension and decimal dilutions.

Presicce D. S., Forleo A., Taurino A. M., Zuppa M., Siciliano P., Laddomada B., Logrieco A., and Visconti A., 2006. Response evaluation of an E-nose towards contaminated wheat by Fusariumpoae fungi. Sensors Actuators B Chemical, 118, 433-438.

Pronyk C., Abramson D., Muir W.E., White N.D.G., 2006. Correlation of total ergosterol levels in stored canola with fungal deterioration. J. Stored Prod. Res., 42,162-172.

Rusinek R. and Kobyłka R., 2014. Experimental study and discrete element method modeling of temperature distributions in rapeseed stored in model bin. J. Stored Product Res., 59, 254-259.

Rusinek R., Molenda M., and Horabik J., 2009. Performance of membrane pressure transducers in granular materials of various particle sizes. Powder Technol., 190, 410-414
Rusinek R., Rybczyński R., Tys J., Gawrysiak-Witulska M., Nogala-Kalucka M., and Siger A., 2012. The process parameters for nontypical seeds during simulated cold deep oil expression. Czech J. Food Sci., 30, 126-134.

Rybiński W., Szot B., Rusinek R., and Bocianowski J., 2009. Estimation of geometric and mechanical properties of seeds of Polish cultivars and lines representing selected species of pulse crops. Int. Agrophys., 23, 257-267.

Sung J., Kim Bum-Keun, Kim Byung-Sam, and Kim Yoonsook, 2014. Mass spectrometry-based electric nose system for assessing rice quality during storage at different temperatures. J. Stored Product Res., 59, 204-208.

Wawrzyniak J., Ryniecki A., and Gawrysiak-Witulska M., 2013. Kinetics of mould growth in the stored barley ecosystem contaminated with Aspergillus westerdijkiae, Penicillium viridicatum and Fusarium poae at $23-30^{\circ} \mathrm{C}$. J. Sci. Food Agric., 93: 895-901.

Wawrzyniak J. and Waśkiewicz A., 2014. Ochratoxin A and citrinin production by Penicillium verrucosum on cereal solid substrates. Food Additives Contaminants: Part A, 31(1), 139-148, DOI 10.1080/19440049.2013.861933

Zhang H. and Wang J., 2007. Detection of age and insect damage incurred by wheat with an electronicnose. J. Stored Products Res., 43, 489-495.

Zhang S., Xie Ch., Hu M., Li H., Bai Y., and Yeng D., 2008. An entire feature extraction method of metal oxide sensors. Sensors and Actuators B: Chemical, 132, 81-89.

Zhou B., Wang J., and Qi J., 2012. Identifications of different wheat seeds by electronic nose. Int. Agrophys., 26, 413-418. 